\title{
MRI-based radiomics to predict lipomatous soft tissue tumors malignancy: a pilot study
}



Benjamin Leporq ${ }^{1 *} \mathbb{D}$, Amine Bouhamama², Frank Pilleul ${ }^{1,2}$, Fabrice Lame $^{2}$, Catherine Bihane ${ }^{2}$, Michael Sdika ${ }^{1}$, Jean-Yves Blay ${ }^{3}$ and Olivier Beuf ${ }^{1}$

\begin{abstract}
Objectives: To develop and validate a MRI-based radiomic method to predict malignancies in lipomatous soft tissue tumors.

Methods: This retrospective study searched in the database of our pathology department, data from patients with lipomatous soft tissue tumors, with histology and gadolinium-contrast enhanced $T_{1}$ w MR images, obtained from 56 centers with non-uniform protocols. For each tumor, 87 radiomic features were extracted by two independent observers to evaluate the inter-observer reproducibility. A reduction of learning base dimension was performed from reproducibility and relevancy criteria. A model was subsequently prototyped using a linear support vector machine to predict malignant lesions.

Results: Eighty-one subjects with lipomatous soft tissue tumors including 40 lipomas and 41 atypical lipomatous tumors or well-differentiated liposarcomas with fat-suppressed $T_{1} w$ contrast enhanced MR images available were retrospectively enrolled. Based on a Pearson's correlation coefficient threshold at $0.8,55$ out of 87 (63.2\%) radiomic features were considered reproducible. Further introduction of relevancy finally selected 35 radiomic features to be integrated in the model. To predict malignant tumors, model diagnostic performances were as follow: AUROC = 0.96 ; sensitivity $=100 \%$; specificity $=90 \%$; positive predictive value $=90.9 \%$; negative predictive value $=100 \%$ and overall accuracy $=95.0 \%$.
\end{abstract}

Conclusion: This work demonstrates that radiomics allows to predict malignancy in soft tissue lipomatous tumors with routinely used MR acquisition in clinical oncology. These encouraging results need to be further confirmed in an external validation population.

Keywords: Magnetic resonance imaging, Radiomic, Liposarcoma

\section{Introduction}

Lipomatous soft tissue tumors are rare tumors arising from benign (such as lipomas) or malignant (liposarcomas) mesenchymal tissue proliferation. Liposarcomas constitute the second most common subgroup of soft tissue sarcomas in adults, with a peak incidence among patients between the fifth and seventh decade (10-35\%)

\footnotetext{
* Correspondence: benjamin.leporq2@gmail.com

'Univ Lyon, INSA-Lyon, Université Claude Bernard Lyon 1, UJM-Saint Etienne, CNRS, Inserm, CREATIS UMR 5220, Villeurbanne, France

Full list of author information is available at the end of the article
}

[1, 2]. The World Health Organization defines four histologic liposarcoma subtypes: well differentiated (WDL), myxoid (including myxoid with round cell component), dedifferentiated, and pleomorphic liposarcoma [3]. Benign lipomas are treated with marginal resection or simple medical follow-up, borderline lesions such as well-differentiated liposarcomas and atypical lipomatous tumors (ALT) require a complete resection to prevent local recurrence and potential dedifferentiation [1, 4]. High-grade liposarcomas may require a multimodal therapy approach according to the aggressiveness of the tumor with large resection and

(c) The Author(s). 2020 Open Access This article is licensed under a Creative Commons Attribution 4.0 International License, which permits use, sharing, adaptation, distribution and reproduction in any medium or format, as long as you give appropriate credit to the original author(s) and the source, provide a link to the Creative Commons licence, and indicate if changes were made. The images or other third party material in this article are included in the article's Creative Commons licence, unless indicated otherwise in a credit line to the material. If material is not included in the article's Creative Commons licence and your intended use is not permitted by statutory regulation or exceeds the permitted use, you will need to obtain permission directly from the copyright holder. To view a copy of this licence, visit http://creativecommons.org/licenses/by/4.0/ The Creative Commons Public Domain Dedication waiver (http://creativecommons.org/publicdomain/zero/1.0/) applies to the data made available in this article, unless otherwise stated in a credit line to the data. 
concomitant chemotherapy and/or radiotherapy [1, 4]. Therefore, noninvasive diagnosis to differentiate benign lipomas from malignant lipomatous soft tissue tumors is crucial to guide the therapeutic strategy. Histology is the gold standard for the diagnosis of lipomatous soft tissue tumors. Nevertheless, specific imaging methods should be considered prior to conducting invasive procedures potentially risky, and preventing unnecessary patient exposure to expensive procedures. These ethical and clinical challenges related to biopsy-based assays can be addressed using medical imaging, routinely used for cancer diagnosis and staging in clinical oncology. However, tumor heterogeneity results in wide range of imaging appearance and reduces the performance of conventional imaging features to efficiently distinguish benign from malignant forms of lipomatous soft-tissue tumors. One of the greatest dilemma for a pathologist lies in differentiating lipoma from WDL or ALT. Brisson and colleagues reported a positive predictive value of $47 \%$ in the diagnosis of ALT/WDL using Magnetic Resonance Imaging (MRI) because of morphological overlap with many benign lipoma variants [5]. Therefore, many biopsies for benign lesions could be avoided and more specific methods are needed to enhance diagnosis.

In the past decade, the field of medical image analysis with an extensive amount of available data is evolving rapidly and resulted in the emergence of radiomics, a discipline deriving imaging features into mineable data (the radiome) for decision support. Radiomics aims to uncover tumor characteristics from different microscopic (genetic, molecular, cellular, histologic) to macroscopic scale [6-8]. The necessary condition is that the images used for radiome extraction (the radiomic fingerprint) translate the underlying pathological mechanisms. We aim to develop and validate a MRI-based radiomic method to specifically distinguish benign lipomas from malignant ALT or WDL.

\section{Patients and method Patients}

This retrospective study searched the database of the Pathology department at our comprehensive cancer center to identify patients with lipomatous soft tissue tumors, with histology and gadolinium-contrast enhanced $\mathrm{T}_{1} \mathrm{w}$ MR images available. The study was approved by our institutional review board and the requirement to obtain informed consent was waived.

\section{MRI data}

MR images were collected from 56 different centers with non-uniform protocols and centralized in the Picture Archiving and Communication System (PACS) of our cancer center. Acquisition were performed at three different fields $(1.0 \mathrm{~T}, 1.5 \mathrm{~T}$, and $3.0 \mathrm{~T})$ with $18 \mathrm{MR}$ systems commercialized by four vendors (Siemens Healthineers, Erlangen, Germany, 39.5\%; General Electric Healthcare,
Milwaukee, WI, USA, 33.3\%; Philips Healthcare, Best, The Netherlands, 24.6\%; and Toshiba medical systems corporation, Otawara, Tochigi, Japan, 2.6\%). Image acquisitions were mainly acquired using a $2 \mathrm{D}$ fast spinecho sequence $(53.3 \%$ with a fat saturation and $11.7 \%$ with a fat-water decomposition) (65\%), a 3D isotropic fast spin-echo sequence (4.9\%), a 3D gradient echo sequence $(16.5 \%$ with a fat saturation and $13.6 \%$ with a fat-water decomposition) and in $35 \%$ of cases with. Mean pixel size was $0.81^{2} \pm 0.29^{2} \mathrm{~mm}^{2}\left(0.37^{2}-1.75^{2}\right)$.

\section{Images processing}

Images were automatically loaded in in-house software developed on Matlab R2017a (The MathWorks, Natick, USA). To evaluate the inter-observer reproducibility, the tumor was manually segmented by two independent observers blinded to histology: an experimented physicist (BL, 13-year experience in MR imaging) and an experimented radiographer (CB, 19-year experience in MR imaging). Tumor segmentation in two dimensions were performed. The slice level with the longest tumor diameter was selected.

Tumor mask was next applied on fat-suppressed enhanced MR images and 87 radiomic features were extracted. They included size, shape, intensity distribution, image domain, and frequency domain textures features. The full list of features is summarized in Fig.1.

Size and shape features were directly extracted from the binary mask. Intensity distribution features were extracted from masked MR images without normalization or filtering of voxel intensities, and from the histogram built with 256 bins. Before the extraction of texture features, images gray levels were discretized in reduced number of gray levels. This operation was done using an equal probability algorithm to define decision thresholds for the volume in order to reach the same number of voxels for a given reconstructed level in the quantized volume, for all gray levels. Images were discretized in 8, 16, 24, 32, 40, 48 and 64 Gy levels and for each discretization level four matrices were built: Gray-level co-occurrence matrix (GLCM), Gray-level run length matrix (GLRLM), Gray-level size zone matrix (GLSZM) and Neighborhood Gray Tone Difference Matrix ((NGTDM) for characteristics extraction (Table.1) as previously reported [9-15]. Frequency domain-based texture features were extracted using a Gabor filtering. GLCM and GLRLM were computed for 4 directions $\left(0^{\circ}, 45^{\circ}, 90^{\circ}\right.$, and $\left.135^{\circ}\right)$ with an offset of 1 pixel. For GLSZM and NGTDM, a 26 pixel connectivity was used. For Gabor filtering, 5 scales, 6 orientations, and a minimal wavelength of 3 were used (Fig.1).

\section{Evaluation of inter-observer variability and effect of gray level discretization}

The inter-observer reproducibility was analyzed using regression models. Pearson's correlation coefficient (r), 


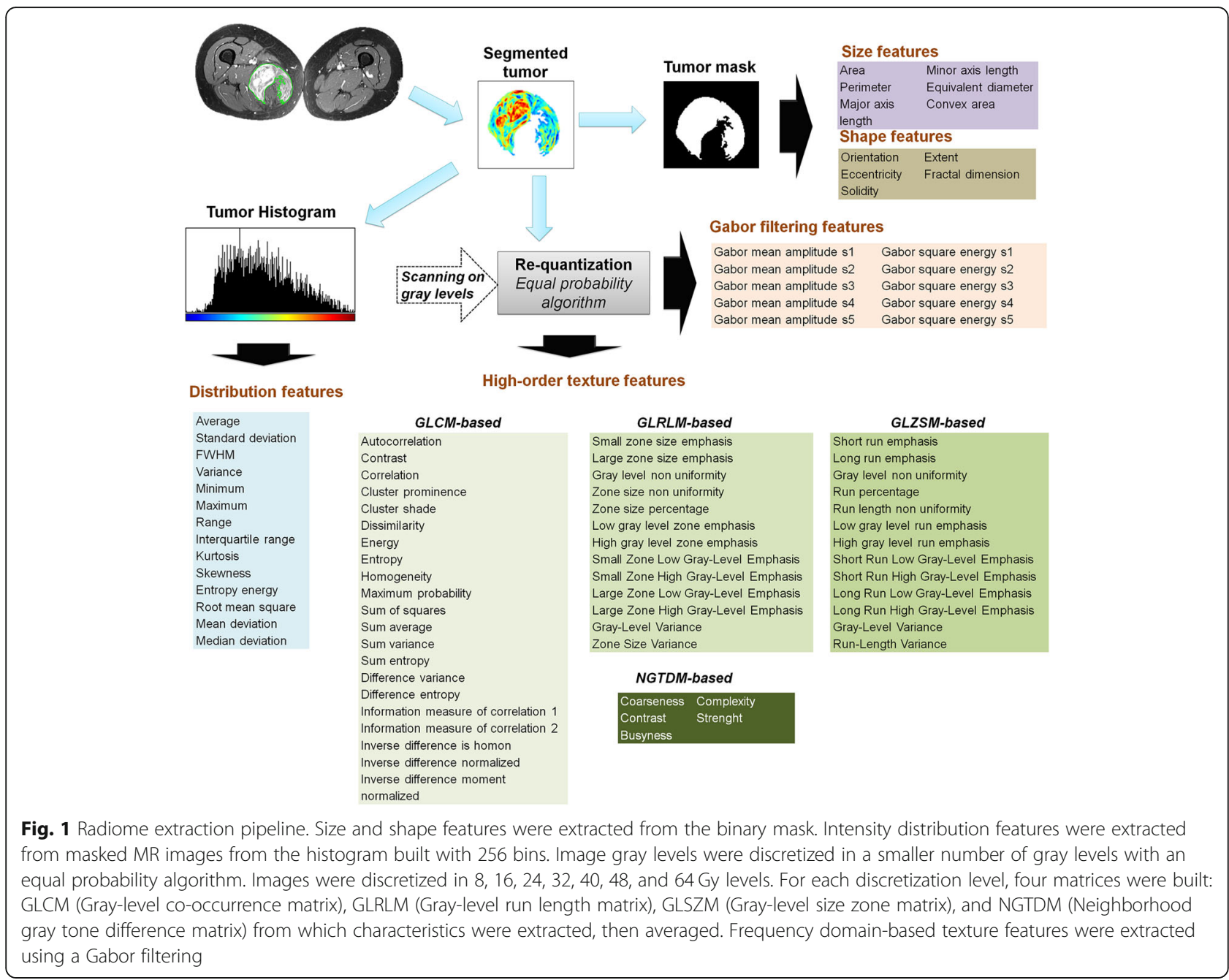

coefficient of determination $\left(\mathrm{R}^{2}\right)$, and Spearman's rankorder correlation coefficient $(\rho)$ were computed.

The effect of the number of gray levels for image discretization was studied by computing the coefficient of variation $(\mathrm{CV})$ for each image domain texture features from values issued from each gray level discretization (i.e. $8,16,24,32,48$, and 64 ) in 10 subjects per group randomly chosen. Next, feature-by-feature, the average $\mathrm{CV}$ on all tested subjects was determined.CV-values from 0 to $5 \%$ were assimilated as an absence of variation, and $\mathrm{CV}$-values from 5 to $25 \%$ were considered as an acceptable variation. $\mathrm{CV}$-values higher than $25 \%$ indicated unacceptable variations.

\section{Prediction of malignant lipomatous tumors}

To predict malignant tumors, a classification model was built using supervised machine learning. The first step reduced the initial set of features to minimize the

Table 1 Linear regression parameters (Pearson correlation coefficient ( $r$ ), coefficient of determination $\left(R^{2}\right)$ and Spearman's rank-order coefficient $(\rho)$ ) computed to evaluate the inter-observer variability according to the radiomics features family

\begin{tabular}{lllllllll}
\hline & Size & Shape & $\begin{array}{l}\text { Intensity } \\
\text { distribution }\end{array}$ & GLCM & GLRLM & GLZSM & NGTDM & Gabor filtering \\
\hline $\mathbf{r}$ & $0.89 \pm 0.04$ & $0.58 \pm 0.20$ & $0.90 \pm 0.16$ & $0.77 \pm 0.08$ & $0.76 \pm 0.13$ & $0.77 \pm 0.17$ & $0.82 \pm 0.08$ & $0.98 \pm 0.02$ \\
& $(0.84-0.93)$ & $(0.27-0.89)$ & $(0.49-0.99)$ & $(0.59-0.86)$ & $(0.57-0.95)$ & $(0.49-0.99)$ & $(0.73-0.93)$ & $(0.96-0.997)$ \\
$\mathbf{R}^{2}$ & $0.80 \pm 0.08$ & $0.38 \pm 0.24$ & $0.83 \pm 0.24$ & $0.6 \pm 0.12$ & $0.6 \pm 0.2$ & $0.62 \pm 0.26$ & $0.67 \pm 0.13$ & $0.96 \pm 0.04$ \\
& $(0.70-0.86)$ & $(0.07-0.79)$ & $(0.24-0.98)$ & $(0.35-0.74)$ & $(0.33-0.89)$ & $(0.24-0.99)$ & $(0.54-0.86)$ & $(0.92-0.99)$ \\
$\boldsymbol{\rho}$ & $0.92 \pm 0.01$ & $0.62 \pm 0.17$ & $0.86 \pm 0.06$ & $0.77 \pm 0.04$ & $0.74 \pm 0.06$ & $0.74 \pm 0.01$ & $0.87 \pm 0.06$ & $0.88 \pm 0.02$ \\
& $(0.90-0.93)$ & $(0.46-0.93)$ & $(0.72-0.92)$ & $(0.68-0.81)$ & $(0.57-0.81)$ & $(0.53-0.84)$ & $(0.77-0.92)$ & $(0.85-0.91)$ \\
\hline
\end{tabular}

Values reported are mean \pm standard deviation (ranges) 
problem of overfitting and created another set of relevant features in term of relevancy and inter-observer reproducibility through filtering with a double thresholding on t-test $p$-value and Pearson's correlation coefficient, previously computed through reproducibility study. Threshold-values were $\mathrm{p}$-value smaller than 0.2 , and Pearson's correlation coefficient higher than 0.8 . The threshold for $p$-value was chosen as a compromise between relevancy and stringency faced with dimensionality reduction to be consistent with observations size.

The second step performed a prototyping of classification model with a support vector machine as a classifier with a linear kernel. Before training, data were centered at their mean and scaled to have unit standard deviation. Support vector computation and hyperplane separation were done using a sequential minimal optimization. The classes were well balanced; a unit box constraint was used for soft margin. To evaluate the overfitting, cross validation used the holdout method (75\% of data were used for training set and 25\% for test set). Similar ratio between benign and malignant lesions was used in both dataset (around 50 vs. $50 \%$ in training and test set).

\section{Effect of MR scanner vendor on features}

The effect of MR vendors on the set of texture features integrated in the model was investigated. For each group, features were stratified by MR vendors and compared using the one-way ANOVA approach with Bonferroni's multiple post-test comparisons.

\section{Results}

\section{Patients}

A total of 81 patients with lipomatous soft tissue tumors were enrolled in this study, 40 lipomas and 41 -differentiated liposarcomas and atypical lipomatous tumors.

\section{Inter-observer reproducibility and effect of number of gray level discretization}

For the whole set of radiomic features, the average Pearson's correlation coefficient was $0.81 \pm 0.15$ [0.27-0.99]; Determination and Spearman $\rho$ average coefficients were $0.68 \pm 0.23$ [0.07-0.99], and $0.79 \pm 0.10$ [0.46-0.93], respectively. Based on a threshold at 0.8 for the Pearson's correlation coefficient, 55 (63.2\%) of 87 radiomic features were considered reproducible. Stratified by feature family, shape features were the least reproducible $(r=$ $0.58 \pm 0.20 ; R^{2}=0.38 \pm 0.24 ; \rho=0.62 \pm 0.17 ; 17 \%$ of features considered reproducible). Gabor filtering features were the most reproducible $\left(r=0.98 \pm 0.02 ; R^{2}=0.96 \pm\right.$ $0.04 ; \rho=0.88 \pm 0.02$; $100 \%$ of features considered reproducible). Results for all features families are summarized in Table 1.

Based on CV-values, the number of gray level impacted $40(76.9 \%)$ of 52 images domain based texture features. Twelve (23.1\%) out of 52 features had acceptable variation including 5 out of 12 features $(41.6 \%)$ with a CV smaller than $5 \%$, considered as totally not impacted by gray level discretization. These features were the following: "Correlation", "Information measure of correlation", "Inverse difference normalized", "Inverse difference moment normalized", and "Coarseness". By texture family, the second order texture features (GLCM -based) were the least impacted ( $\mathrm{CV}=59.8 \pm 50.6 \%$ [0.37-154\%]). High order texture features were more impacted: $\mathrm{CV}=69.0 \pm 38.2 \%$ [10.8-132\%]; $\mathrm{CV}=95.6 \pm$ $58.6 \%$ [18.5-209\%]; $C V=89.1 \pm 61.1 \%[4.72-158 \%]$ for GLRLM, GLSZM and NGTDM texture features respectively.

\section{Prediction of malignant lipomatous tumors}

Based on t-test $p$-value, out of 87 features, 64 (73.6\%) were included in the reduced feature set. Size features were the most relevant (100\% of relevant features), intensity distribution were the worst relevant $(28.6 \%$ of relevant features). Results for each family are summarized in Table 2. After combination with reproducibility criterion, the radiome was finally reduced to $35(40.2 \%)$ features. Details on preselected features were provided in Fig. 2. An example illustrating radiomic features variations according to benign or malignant status is provided in Fig. 3. To distinguish benign from malignant tumors, model diagnostic performances were as follow: AUROC $=0.96$; sensitivity $=100 \% \quad(95 \% \mathrm{CI} \quad 100-100 \%)$; specificity $=90 \%(95 \%$ CI $71.4-108 \%)$; positive predictive value $=90.9 \%$ (95\%CI $73.9-108 \%)$; negative predictive value $=100 \%(95 \%$ CI $100-100 \%)$, and overall accuracy $=$ 95.0\% (95\%CI 85.5-105\%).

\section{Effect of MR scanner vendor on features}

The benign group showed no significant differences according to the different manufacturers. In the malignant group, 3 GLCM texture-based features displayed significant differences ("Contrast", "Dissimilarity", and

Table 2 Percentage of relevant features to discriminate benign from malignant lipomatous tumors

\begin{tabular}{ll}
\hline & Benign vs. Malignant \\
\hline Size & $100 \%(6 / 6)$ \\
Shape & $80.0 \%(4 / 5)$ \\
Intensity distribution & $28.6 \%(4 / 14)$ \\
GLCM & $95.2 \%(20 / 21)$ \\
GLRLM & $92.3 \%(12 / 13)$ \\
GLSZM & $69.2 \%(9 / 13)$ \\
NGTDM & $80.0 \%(4 / 5)$ \\
Gabor filtering & $50.0 \%(5 / 10)$ \\
\hline Attest $p<0.2$ was conside
\end{tabular}

A t test $p<0.2$ was considered for relevancy 


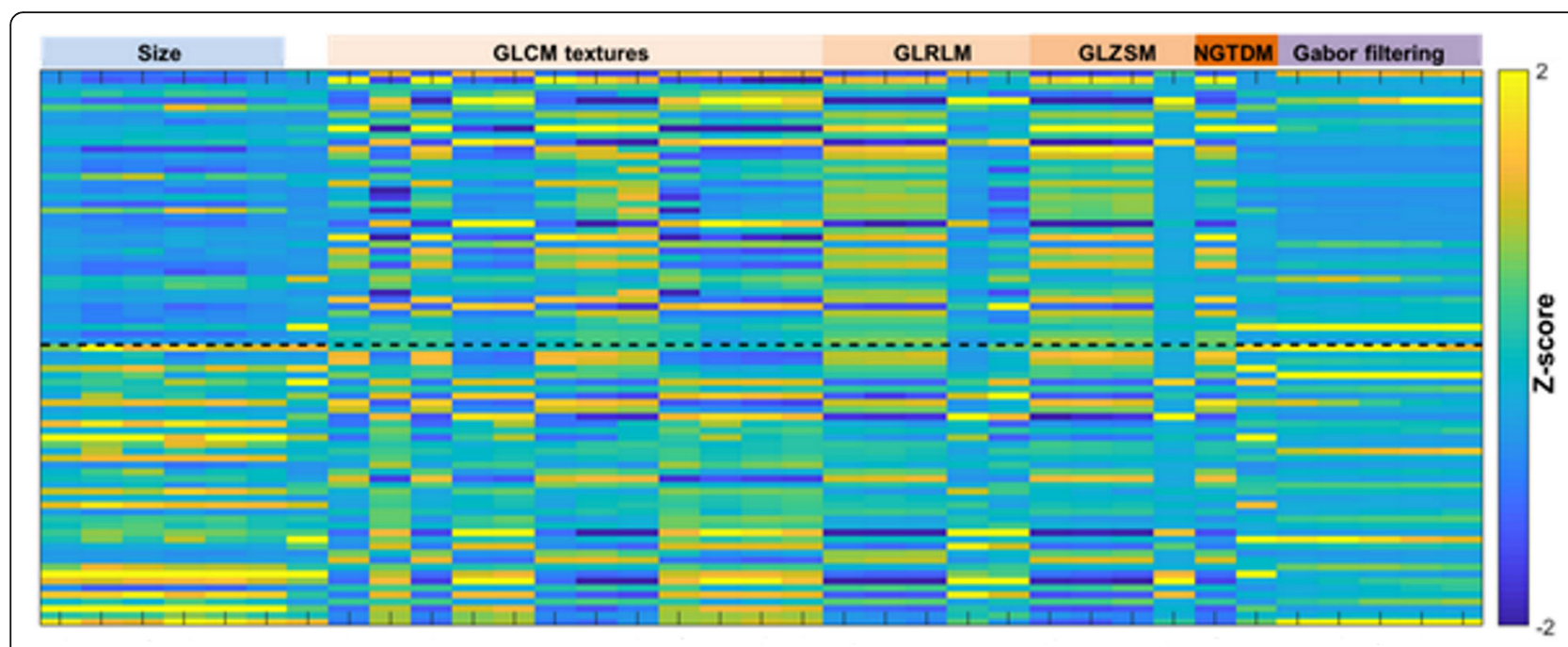

Fig. 2 Heatmap representing the reduced learning base after features filtering from reproducibility and relevancy criterion. From the initial feature set, only 35 features were integrated. Size and high order texture features were largely integrated whereas shape and intensity distribution features were not integrated due to poor reproducibility and relevancy, respectively. Black dash line represents the limit between the two classes

"Difference variance"). These differences were systematically identified between General Electric and Philips MR systems. No significant differences in pixel sizes were observed according to the different MR vendors and between the groups, regardless of MR vendors.

\section{Discussion}

This work showed that routine MRI acquisition allows to evaluate lipomatous soft-tissue malignancy in clinical practice with promising diagnostic performances. Our radiomic analysis used fat-suppressed contrast enhanced

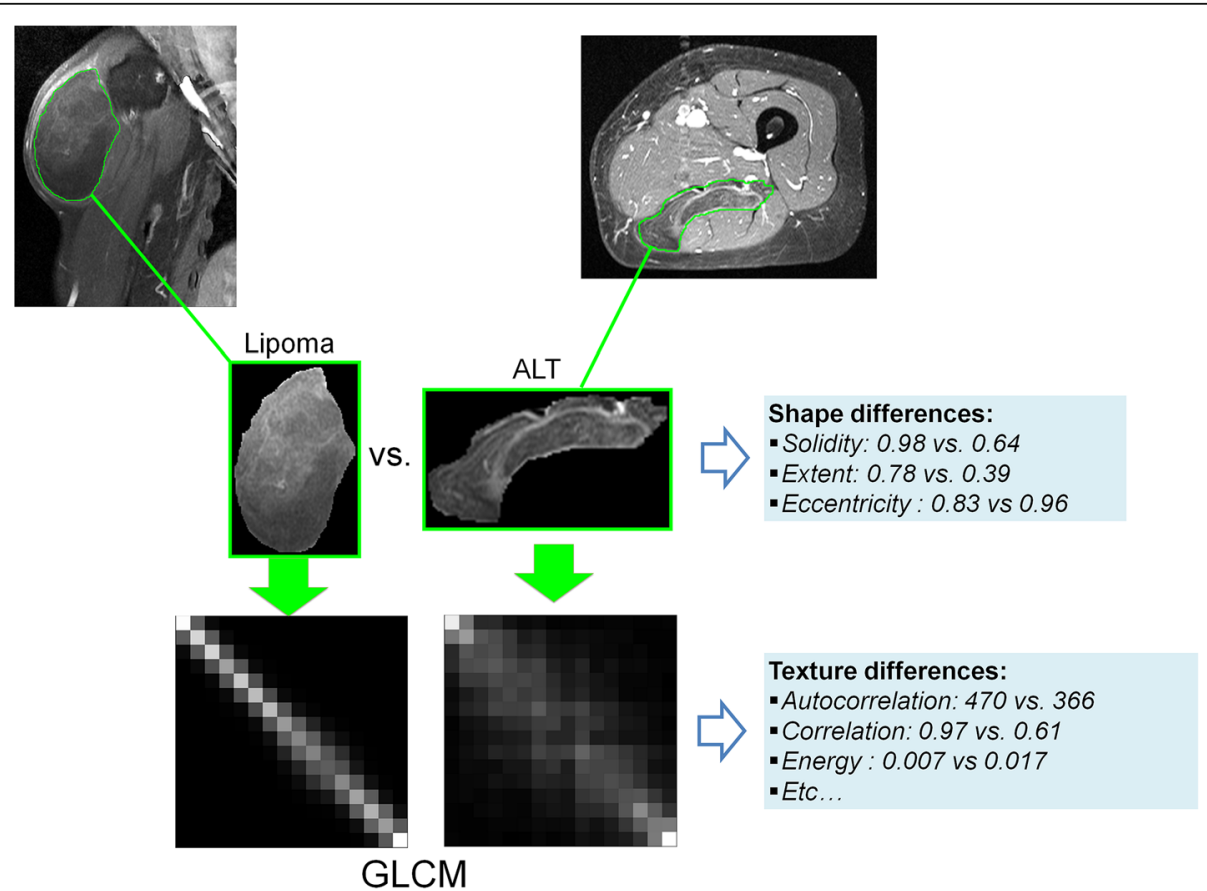

Fig. 3 Malignant ALT (Atypical Lipomatous Tumors) display visual differences in shape comparison with lipoma. These differences were quantified by shape radiomics features (such as solidity, extent, and eccentricity), and expressed in the radiome. Tumor enhancements display different texture which can be recorded by the GLCM (Gray-level co-occurrence matrix); quantified using GLCM-based descriptors and expressed in the radiome 
$\mathrm{T}_{1}$-weighted images collected from 56 institutions. Our aim was to differentiate lipomas from WDL/ALT, considered as the most challenging issue in clinical practice. To our knowledge, this study is the first to report MRbased radiomic results to evaluate lipomatous tumor malignancy. According to these results, all lesions were detected with $100 \%$ sensitivity and a good specificity (90\%) suggesting that radiomics may help to decrease the number of unnecessary biopsy. This issue is central for ethical and healthcare cost-effective purpose.

The gadolinium uptake used a single radiomic fingerprint. The radiome only gathered information translating vascular and necrosis processes. Oedema and fat content have also been described to discriminate lipomas from liposarcomas [9, 16-19]. Therefore, the combined use of suppressed $\mathrm{T}_{2}$-weighted $\mathrm{MR}$ imaging and/or no fatsuppressed acquisition could still improve diagnostic performances reported in the present study. However, such MRI acquisitions are not systematically required in clinical protocols.

Size features were found relevant to predict malignant lipomatous tumors and were largely integrated in the models. This illustrated that tumor size may help to characterize the malignancy as reported in previous works based on conventional imaging characteristic $[16,18-24]$. Texture features (particularly GLCMbased) were largely reported as relevant features, whereas intensity distribution features were not strongly appropriate, and demonstrate that the spatial heterogeneity of gadolinium enhancement is more discriminant than its intensity to isolate benign lesion. This could be explained by the presence of a structured capillary network and/or nodular septa in malignant lesions. This is also consistent with clinical practice based on visual characterization of tumor enhancement homogeneity and the presence of thick and nodular septa as key characteristics $[5,19]$. We reported poor inter-observer reproducibility, shape features were consequently not integrated, despite a good relevancy. Shape differences observed here may also reflect excessive cell proliferation in malignant tumors [25]. Tumor shape belong to key characteristics visually assessed in clinical practice $[18,19]$.

The implementation of multivariate scores issued from the combination of conventional MR imaging features to distinguish benign lipomas from ALT alone or ALT/ WDL have been previously reported $[19,20]$. However, features were subjective and only qualitative. These scores may be strongly exposed to inter-observer variability and may depend on the experience of the radiologist. However, such previous results clearly demonstrated the interest of combining different quantitative features expressing subjective characteristics, such as radiomics does.

Our results suggested that radiomic features are also exposed to inter-observer variability. The segmentation step was strongly assumed to introduce these discrepancies as the single non-automated step in the processing pipeline. Based on the Pearson's correlation coefficient, only $63 \%$ of features were found to be reproducible. Features were not equally impacted. Shape features were the most impacted, which may be explained by an observer disagreement in the choice of slice level for tumor contouring. Size and Gabor filtering texture features were less impacted with $100 \%$ of features considered as reproducible. To prospectively classify lesion groups, the inherent observer variability in the data mining step should be carefully considered. We subsequently proposed to discard features from the initial radiome by thresholding on Pearson's correlation coefficient and implement the model from a reduced data set. We have also investigated the effect of the number of gray level for image discretization on texture features stability and showed a strong impact of discretization with $77 \%$ of features impacted. GLCM based-texture seem to be less impacted than higher order features based on GLRLM, GLZSM and NGTDM features, as previously reported [26, 27]. This result suggested that method standardization for feature extraction is mandatory for prospective use in multicentric trials or for retrospective use of annotated data set. In parallel, selection of number of gray level impacted the feature relevancy for classifying. However, this hypothesis was not explored neither evaluated in this study. Therefore, we have proposed to introduce the number of gray level used for the discretization in a second dimension by quantifying texture features on a discrete range of gray level (between 8 to 64), and used the average as final values for each texture features. Out of the integrated features, a slight vendor effect was observed, mainly between General electric and Philips MR systems and limited for GLCM texture-based features. Other factors, regardless MR vendors could introduce pixel value differences such as the repetition time, the injected dose of gadolinium, the delay between injection and acquisition, and the fat saturation procedure. These outcomes were not directly investigated, but the model prototyped from these heterogeneous data has provided indirect evidence on these issues.

This study has several limitations. First, image resolution has also been reported to impact features stability and we did not use a resampling procedure to normalize pixel size in this work. However, pixel size displayed no significant differences between groups.

Second, although internal cross-validation on test and application data was used to build the model, no external validation on independent population was performed. In this pilot study, the multicenter multi-vendor aspect in the database for subsequently integration of the maximum of acquisition heterogeneities in our model has been preferred. As a consequence, these encouraging results need to be further confirmed on 
multicenter prospective or applied to existing population to rigorously verify the impact of MR-vendor and MR protocol on classification performances.

Third, segmentation was performed only in two dimensions. The rationale supporting two-dimension segmentation rather than a three-dimension was to simplify the annotation step. This choice may have enhanced feature inter-observer variability, particularly for shape features. In addition, an inherent risk of sampling effect may have been subsequently generated. These issues need to be further investigated.

\section{Conclusion}

To conclude, this work demonstrates that radiomics allows to predict malignancy in soft tissue lipomatous tumors with routine MR acquisition in clinical oncology. These encouraging results need to be further confirmed in a validation population.

\section{Abbreviations}

WDL: Well-Differentiated Lipomas; ALT: Atypical Lipomatous Tumors; MRI: Magnetic Resonance Imaging; GLCM: Gray-Level Co-occurrence Matrix); GLRLM: Gray-Level Run Length Matrix); GLSZM: Gray-Level Size Zone Matrix); NGTDM: Neighborhood Gray Tone Difference Matrix); CV: Coefficient of Variation; AUROC: Area Under the Receiver Operating Curve

\section{Acknowledgements}

This work was performed within the framework of the SIRIC LyriCAN grant INCa_INSERM_DGOS_12563 and LABEX PRIMES (ANR-11-LABX-0063), program "Investissements d'Avenir" (ANR-11-IDEX-0007).

The authors thank Sophie Darnis (Centre Léon Bérard) for medical editorial assistance with this manuscript.

\section{Availability of supporting data}

Authors confirm the availability of data and material. Please contact author for data requests.

\section{Authors' contributions}

Benjamin Leporq: Acquisition of data, analysis and interpretation of data, drafting of manuscript and critical revision. Amine Bouhamama: Study conception and design, analysis and interpretation of data, drafting of manuscript and critical revision. Fabrice Lame: Study conception and design and critical revision. Catherine Bihane: Acquisition of data. Michael Sdika: Critical revision. Jean-Yves Blay: Critical revision. Olivier Beuf: Critical revision. Frank Pilleul: Study conception and design, Critical revision.

\section{Funding}

Authors did not receive any source of funding for this work.

\section{Ethics approval and consent to participate}

This retrospective study was approved by our Institutional Review Board (CPP Lyon-Sud-Est IV Centre Léon Bérard, N N IRB: IRB00010619) with a waiver of informed consent, with any overlap in subjects from prior publications to disclose.

\section{Consent for publication}

Not applicable.

\section{Competing interests}

The authors declare that they have no competing interests.

\section{Author details}

'Univ Lyon, INSA-Lyon, Université Claude Bernard Lyon 1, UJM-Saint Etienne, CNRS, Inserm, CREATIS UMR 5220, Villeurbanne, France. ${ }^{2}$ Department of Radiology, CRLCC Léon Berard, Lyon, France. ${ }^{3}$ Department of Oncology, CRLCC Léon Berard, Lyon, France.
Received: 2 November 2018 Accepted: 1 October 2020

Published online: 28 October 2020

\section{References}

1. Murphey MD, Arcara LK, Fanburg-Smith J. From the archives of the AFIP: imaging of musculoskeletal liposarcoma with radiologic-pathologic correlation. Radiographics. 2005;25:1371-95.

2. O'Regan KN, Jagannathan J, Krajewski K, et al. Imaging of liposarcoma: classification, patterns of tumor recurrence and response treatment. Am J Roentgenol. 2011;97:37-43.

3. Fletcher C. WHO classification of tumours of soft tissue and bone (2013). IARC:19-46.

4. Murphey MD, Carroll JF, Flemming DJ, Pope TL, Gannon FH, Kransdorf MJ. From the archives of the AFIP: benignmusculoskeletal lipomatous lesions. Radiographics. 2004;24:1433-66.

5. Brisson M, Kashima T, Delaney D, et al. MRI characteristics of lipoma and atypical lipomatous tumor/well-differentiated liposarcoma: retrospective comparison with histology and MDM2 gene amplification. Skelet Radiol. 2013:42:635-47.

6. Segal $E$, Sirlin $C B$, Ooi $C$, et al. Decoding global gene expression programs in liver cancer by noninvasive imaging. Nat Biotechnol. 2007;25:675-80.

7. Diehn M, Nardini C, Wang DS, et al. Identification of noninvasive imaging surrogates for brain tumor gene-expression modules. Proc. Natl Acad. Sci USA. 2008;105:5213-8.

8. Aerts HJ, Velazquez ER, Leijnaar R, et al. Decoding tumour phenotype by noninvasive imaging using a quantitative radiomics approach Nat. Commun. 2014,5:4006.

9. Thibault G, Fertil B, Navarro C, et al. Texture indexes and gray level size zone matrix: application to cell nuclei classification. Patt Recogn Inform Process. 2009:140-5.

10. Galloway MM. Texture analysis using gray level run lengths. Comput. Graph. Imag Process. 1975;4:172-9.

11. Haralick RM, Shanmugam K, Dinstein I. Textural features for image Classification IEEE trans. Syst. Man Cybern. 1973;3:610-21.

12. Amadasun M, King R. Textural features corresponding to textural properties. IEEE Transact Syst Man Cybernetics. 1989;19:1264-74.

13. Chu A, Sehgal C, Greenleaf J. Use of gray value distribution of run lengths for texture analysis. Pattern Recogn Lett. 1990;11:415-9.

14. Dasarathy $B$, Holder E. Image characterizations based on joint gray level-run length distributions. Pattern Recogn Lett. 1991;12:497-502.

15. Kickingereder $\mathrm{P}$, Burth $\mathrm{S}$, Wick $\mathrm{A}$, et al. Radiomic profiling of glioblastoma: identifying an imaging predictor of patient survival with improved performance over established clinical and radiologic risk models. Radiology. 2016;280:880-9.

16. El Ouni F, Jemni H, Trabelsi A, et al. (2010). Liposarcoma of the extremities: MR imaging features and their correlation with pathologic data. Orthop Traumatol Surg Res. 2010;96:876-83.

17. Galant J, Martí-Bonmatí L, Sáez F, Soler R, Alcalá-Santaella R, Navarro M. The value of fat-suppressed T2 or STIR sequences in distinguishing lipoma from well-differentiated liposarcoma. Eur Radiol. 2003;13:337-43.

18. Jaovisidha S, Suvikapakornkul Y, Woratanarat P, Subhadrabandhu T, Nartthanarung A, Siriwongpairat P. MR imaging of fat containing tumours: the distinction between lipoma and liposarcoma. Singap Med J. 2010;51: 418-23.

19. Wang S, Chan LW, Tang X, et al. A weighted scoring system to differentiate malignant liposarcomas from benign lipomas. J Orthop Surg (Hong Kong). 2016;24:216-21

20. Nagano S, Yokouchi M, Setoguchi T, et al. Differentiation of lipoma and atypical lipomatous tumour by a scoring system: implication of increased vascularity on pathogenesis of liposarcoma. BMC Musculoskelet Disord. 2015;16:36.

21. Gelineck J, Keller J, Jensen OM, Nielsen OS, Christensen T. Evaluation of lipomatous soft tissue tumours by MR imaging. Acta Radiol. 1994;35:367-70.

22. Datir A, James SL, Ali K, Lee J, Ahmad M, Saifuddin A. MRI of soft-tissue masses: the relationship between lesion size, depth, and diagnosis. Clin Radiol. 2008;63:373-8.

23. Coran A, Ortolan P, Attar S, et al. Magnetic resonance imaging assessment of lipomatous soft-tissue tumors. Vivo. 2017;31:387-95.

24. Sung MS, Kang HS, Suh JS, et al. Myxoid liposarcoma: appearance at MR imaging with histologic correlation. Radiographics. 2000;20:1007-19. 
25. Zhu Y, Li H, Guo W, et al. Deciphering genomic underpinnings of quantitative MRI-based radiomic phenotypes of invasive breast carcinoma. Sci Rep. 2015;7(5):17787.

26. Larue RTHM, van Timmeren JE, de Jong EEC, et al. Influence of gray level discretization on radiomic feature stability for different CT scanners, tube currents and slice thicknesses: a comprehensive phantom study. Acta Oncol. 2017:8:1-10

27. Leijenaar RT, Nalbantov G, Carvalho S et al (2015). The effect of SUV discretization in quantitative FDG-PET radiomics: the need for standardized methodology in tumor texture analysis. Sci Rep; 5:5:11075.

\section{Publisher's Note}

Springer Nature remains neutral with regard to jurisdictional claims in published maps and institutional affiliations.

Ready to submit your research? Choose BMC and benefit from:

- fast, convenient online submission

- thorough peer review by experienced researchers in your field

- rapid publication on acceptance

- support for research data, including large and complex data types

- gold Open Access which fosters wider collaboration and increased citations

- maximum visibility for your research: over $100 \mathrm{M}$ website views per year

At BMC, research is always in progress.

Learn more biomedcentral.com/submissions 